\title{
The BaSTI Stellar Evolution Database: models for extremely metal-poor and super-metal-rich stellar populations
}

\author{
Adriano Pietrinferni ${ }^{1}$, Santi Cassisi ${ }^{1}$, Maurizio Salaris ${ }^{2}$, and Sebastian Hidalgo ${ }^{3}$ \\ ${ }^{1}$ INAF - Osservatorio Astronomico di Teramo, via M. Maggini, 64100 Teramo, Italy \\ e-mail: [cassisi;pietrinferni]@oa-teramo.inaf.it \\ 2 Astrophysics Research Institute, Liverpool John Moores University, Liverpool Science Park, 146 Brownlow Hill, IC2 Building, \\ Liverpool L3 5RF, England \\ e-mail: M.Salaris@ljmu.ac.uk \\ 3 Instituto Astrofisico de Canarias, C.lle Via Lactea, sn., 38532 La Laguna (Tenerife), Spain \\ e-mail: shidalgo@iac.es
}

Received 24 May 2013 / Accepted 30 July 2013

\section{ABSTRACT}

\begin{abstract}
We present an extension of the BaSTI (Bag of Stellar Tracks and Isochrones) stellar evolution database to extremely metal-poor $\left(Z=10^{-5}\right)$ and super-metal-rich $(Z=0.05)$ metallicities, with both scaled-solar and $\alpha$-enhanced $([\alpha / \mathrm{Fe}]=0.4)$ heavy element distributions. These new tracks (from the pre-main sequence to the early-asymptotic giant branch phase), horizontal branch models, and isochrones will enable the use of the BaSTI database to study the most metal-poor populations found in Local Group faint dwarf galaxies, and the metal-rich component of the Galactic bulge, for example. An overview of several fundamental predictions of stellar evolution over the full metallicity range of BaSTI is presented, together with comparisons with literature calculations at $Z=10^{-5}$ and $Z=0.05$.
\end{abstract}

Key words. stars: evolution - stars: interiors - stars: late-type - Hertzsprung-Russell and C-M diagrams - stars: low-mass

\section{Introduction}

Libraries of stellar models and isochrones covering wide ranges of age and metallicities are an essential tool to investigate the properties of resolved and unresolved stellar populations. This is the seventh paper in a series devoted to BaSTI (a Bag of Stellar Tracks and Isochrones) $)^{1}$. We aim to create an extended, complete, and up-to-date database of theoretical models and byproducts such as isochrones, luminosity functions (LF), tables of integrated spectra and magnitudes of simple stellar populations, and synthetic colour-magnitude diagrams (CMDs) of simple/composite stellar systems.

The first paper (Pietrinferni et al. 2004; hereafter Paper I) presented scaled-solar stellar evolution models and isochrones, while Pietrinferni et al. (2006; hereafter Paper II) extended the database to $\alpha$-enhanced metal compositions appropriate, for example, for modelling the stellar population of the Galactic halo (Gratton et al. 2004, and references therein). The inclusion of the asymptotic giant branch phase, and then description of the BaSTI synthetic CMD generator (SYNTHETIC_MAN) was included in Cordier et al. (2007; hereafter Paper III), whilst integrated spectra and magnitudes for the whole set of chemical compositions were published by Percival et al. (2009; hereafter Paper IV). Pietrinferni et al. (2009; hereafter Paper V) extended BaSTI to mixtures including the CNONa abundance anticorrelations observed in Galactic globular clusters (GCs), while Salaris et al. (2010; hereafter Paper VI) added to the database white dwarf (WD) cooling sequences and isochrones. As part of our ongoing effort to provide the scientific community with a selfconsistent evolutionary framework for interpreting observations

\footnotetext{
1 http://wWw.oa-teramo.inaf.it/BASTI
}

of a wide range of stellar populations, we now present an extension of the BaSTI archive to both extremely metal-poor and super-metal-rich stellar populations beyond the present BaSTI metallicity range.

Observations of metal-poor candidates in the HK survey (see e.g. Christlieb et al. 2008, and references therein), the Sloan Digital Sky Survey (York et al. 2000), and the SEGUE survey (Yanny et al. 2009) have vastly increased the available sample of Galactic extremely metal-poor stars, with $[\mathrm{Fe} / \mathrm{H}]<-3.5$ (see also Norris et al. 2013). Needless to say, the interpretation of the chemical and evolutionary properties of these stars can provide vital clues to the earliest phases of the formation and evolution of the Galaxy.

At the same time, detailed investigations of the smallest galaxies in the Local Group, the so-called ultra faint dwarfs (Belokurov et al. 2007, 2010; Norris et al. 2010) have shown that these stellar systems host a sizable, if not dominant population of very metal-poor objects. Observations have disclosed the presence of stars with $[\mathrm{Fe} / \mathrm{H}]$ as low as $\approx-3.7$ (see e.g. Fulbright et al. 2004; Norris et al. 2008, 2010). To infer the star formation history of these Milky Way satellites, population synthesis codes have to account for the observed low metallicities.

Extended sets of stellar models for extremely metal-poor stars have been calculated by Cassisi \& Castellani (1993), Cassisi et al. (1996, 1997); these calculations have enabled detailed investigations of the properties of extremely metalpoor objects and primordial stellar populations (Raimondo et al. 2001). However, the significant improvements of the last decade regarding stellar physics inputs (see Cassisi 2005, 2009, 2010, 2012, and references therein) require an updated theoretical framework for these populations. 
At the other extreme of the metallicity spectrum, both spectroscopic and photometric surveys (see Gonzalez et al. 2011, and references therein) show that the Galactic bulge metallicity distribution (peaked at about solar metallicity) displays an extended tail reaching $[\mathrm{Fe} / \mathrm{H}] \sim+1.0$. High-metallicity stars are also an important constituent of elliptical galaxies, and their post-main sequence (post-MS) evolution is considered to be responsible (Greggio \& Renzini 1990) for the observed UV excess, the so called UV-upturn phenomenon (Burstein et al. 1988, and references therein), observed in the spectra of a fraction of elliptical galaxies. From a theoretical point of view, during the last 15 years calculations of very metal-rich models have received increasing attention (see e.g. Bono et al. 1997; Dotter et al. 2007), and we therefore extend BaSTI to cover models at the upper end of the observed cosmic metallicities.

The extension of BaSTI to both extremely metal-poor and super-metal-rich compositions will complete the creation of an updated and self-consistent theoretical evolutionary framework that covers almost the whole metallicity interval spanned by stars in both the Galaxy and extra-galactic stellar systems.

The paper is organized as follows: Sect. 2 summarizes the model physics inputs and describes calculations and main evolutionary properties. Comparisons with models available in literature are discussed in Sect. 3. A summary and final remarks follow in Sect. 4.

\section{The theoretical framework}

To be consistent with the existing BaSTI calculations, we used the same stellar evolution code (see Papers I, II, and III), and input physics of the previous calculations (see Papers I, II, and III) e.g. radiative and electron conduction opacities, equation of state, and nuclear cross sections. Superadiabatic convection is treated according to the Cox \& Giuli (1968) formalism of the mixing length theory (Böhm-Vitense 1958), with the the mixing length value equal to 2.023, as obtained from a calibration of the standard solar model (SSM) ${ }^{2}$. Outer boundary conditions have been computed by integrating the atmospheric layers with the $T(\tau)$ relation by Krishna-Swamy (1966).

All models include mass loss using the Reimers formula (Reimers 1975) with the free parameter $\eta=0.4^{3}$. For consistency with the other computations already available at the BaSTI database, all stellar models presented in this work have been computed by neglecting the occurrence of atomic diffusion.

As for the other calculations already available in the BaSTI library, we computed models with and without convective core overshoot during the H-burning phase (when convective cores are present). We adopted exactly the same extension of the overshooting region as a function of mass that we used in our previous calculations.

The extremely metal-poor chemical compositions presented here have $Y=0.245$ (Cassisi et al. 2003), total metallicity $Z=10^{-5}$, and both a scaled solar metal mixture (as in Paper I we

\footnotetext{
2 The first release of the BaSTI database relied on the low-temperature opacities by Alexander \& Ferguson (1994) that required a mixing length value equal to 1.913 for the SSM. When the new low- $T$ opacities by Ferguson et al. (2005) were released, they were adopted to recompute the whole BaSTI archive; in this case the SSM calibration required a mixing length equal to 2.023 .

3 The BaSTI archive provides calculations for two different values for the free parameter $\eta$, namely 0.2 and 0.4 . Our preferred value for population synthesis models is $\eta=0.4$. We also noticed that this value has become a standard choice among BaSTI users, and therefore here we decided to neglect the $\eta=0.2$ computations.
}

rely on the Grevesse \& Noels 1993 mixture) and an $\alpha$-enhanced one (see Paper II for more details). The scaled solar calculations correspond to $[\mathrm{Fe} / \mathrm{H}]=-3.27$, whilst the $\alpha$-enhanced ones correspond to $[\mathrm{Fe} / \mathrm{H}]=-3.62$.

The super-metal-rich calculations are for $Y=0.316$ (corresponding to $\Delta Y / \Delta Z \sim 1.4$, as in the existing BaSTI calculations) and $Z=0.05$, both scaled solar and $\alpha$-enhanced. These choices correspond to $[\mathrm{Fe} / \mathrm{H}]=+0.51$ (scaled solar) and +0.16 ( $\alpha$-enhanced). The adopted $\Delta Y / \Delta Z \sim 1.4$ for the supersolar regime is corroborated by the good match of BaSTI isochrones to the CMD of the old open cluster NGC $6791([\mathrm{Fe} / \mathrm{H}] \approx 0.4)$ and a derived distance modulus $\left((m-M)_{v}=13.50\right.$; Bedin et al. $2008)$ in perfect agreement with the eclipsing binary distance to the cluster determined by Brogaard et al. (2012).

For each chemical composition we have computed stellar models with mass ranging from $0.50 M_{\odot}$ to $10 M_{\odot}{ }^{4}$. All models, with the exception of those with a MS lifetime longer than the Hubble time for which the computation has been stopped at the central $\mathrm{H}$ exhaustion, have been computed from the pre-MS until the beginning of the thermal pulse stage along the asymptotic giant branch. These have been supplemented by an extended set of horizontal branch (HB) calculations, by adopting the He-core mass and envelope $\mathrm{He}$ abundance of a red giant branch (RGB) progenitors whose age at He-ignition is $\approx 13 \mathrm{Gyr}\left(0.8 M_{\odot}\right.$ for $Z=10^{-5}$ and $1.0 M_{\odot}$ for $\left.Z=0.05\right)$. These models allow the synthetic HB populations to be computed with an arbitrary mass distribution. Figure 1 displays the theoretical Hertzsprung-Russell (HR) diagram of selected tracks and isochrones for both metallicities, whilst Fig. 2 shows HB tracks.

The evolutionary tracks have been reduced to the same number of points, to facilitate the computation of isochrones and their use in population synthesis codes, by identifying along each evolutionary track some characteristic homologous points, also known key points (KPs) corresponding to well-defined evolutionary phases. For a careful description of the adopted KPs we refer the reader to Paper II. The whole set of evolutionary computations (except for the additional HB models discussed above) have been used to compute isochrones from $30 \operatorname{Myr}\left(50 \mathrm{Myr}\right.$ for $Z=10^{-5}$ ) to 15 Gyr. Finally, tracks and isochrones have been transformed to various photometric systems (i.e. Johnson-Cousins, ACS, and WFC3 Vegamag) by using the same colour- $T_{\text {eff }}$ transformations and bolometric corrections presented in Papers I and II, and Bedin et al. (2005). These computations are made public at the BaSTI official website ${ }^{5}$.

\subsection{Main evolutionary properties of extremely metal-poor and super-metal-rich stars}

Figure 1 reveals some important evolutionary features of these models related to their extreme metallicities. At fixed initial mass, extremely metal-poor stars are obviously much hotter and brighter than their more metal-rich counterparts. This occurrence, as is well known, is due to the huge dependence of the stellar radiative opacity on the metallicity: the larger the metallicity, the larger the radiative opacity. For an overview across the whole metallicity range spanned by the BaSTI models, Tables 1 and 2 list some relevant evolutionary features for selected models, with $Z$ ranging from $Z=10^{-5}$ to 0.05 .

The strong dependence of the $T_{\text {eff }}$ scale of low-mass models on $Z$, associated with the metallicity dependence of the radiative opacity, is shown in Fig. 3 that compares tracks for the same

\footnotetext{
4 For $Z=10^{-5}$ the most massive stellar model corresponds to $6 M_{\odot}$.

5 http://www.oa-teramo.inaf.it/BASTI
} 
A. Pietrinferni et al.: Extension of the BaSTI model archive
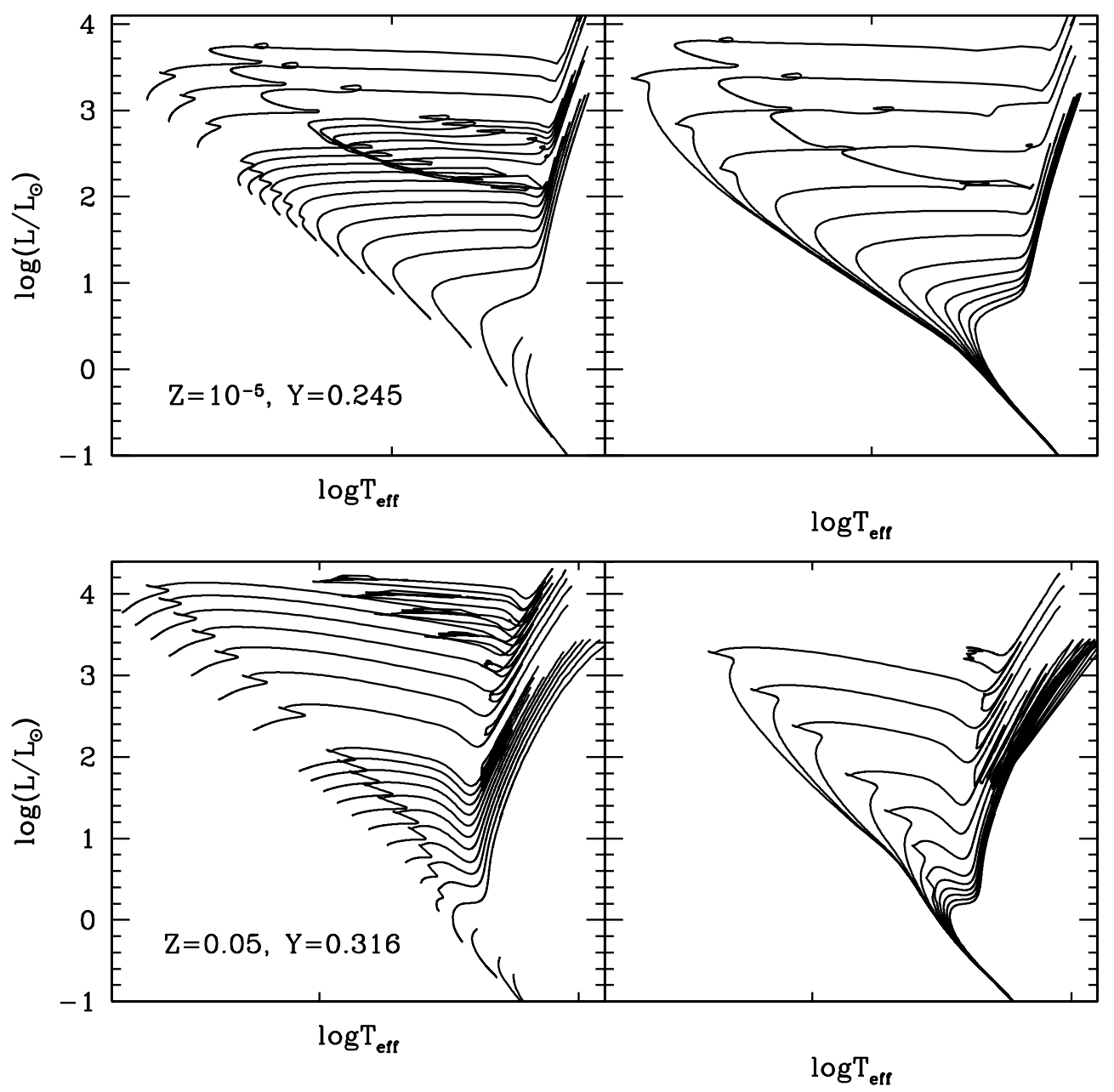

Fig. 1. Upper-left panel: evolutionary tracks of selected stellar models (no convective core overshooting during the H-burning phase) with $Z=10^{-5}, Y=0.245$, and $M / M_{\odot}=0.5,0.6,0.8,1.0,1.2,1.4,1.6,1.8,2.0,2.2,2.4,2.6,2.8,3.0,4.0,5.0,6.0$, and a scaled solar heavy elements mixture. All tracks for low-mass stars (apart from those masses with lifetimes much longer than the Hubble time) are displayed up to the tip of the red giant branch (RGB). Upper-right panel: selected isochrones for the same chemical composition, with ages $\mathrm{t}(\mathrm{Gyr})=0.05,0.10,0.20,0.5,1.0,2.0,4.0,6.0,8.0,10.0,12.0,14.0$. Lower-left panel: as in the upper-left panel, but for $Z=0.05, Y=0.316$. Masses equal to $M / M_{\odot}: 7,8,9,10$ are also shown. Lower-right panel: as in the upper-right panel, but for the metal-rich chemical composition.

mass and all (scaled solar) metallicities available in the BaSTI database. However, when metallicity values go down to values $Z \leq 10^{-4}$, the dependence of the radiative opacity on the metal content vanishes, as demonstrated by the two tracks for $Z=10^{-4}$ and $Z=10^{-5}$ that overlap almost perfectly along the MS and the RGB. This agrees with the results by Cassisi \& Castellani (1993, and references therein).

Something similar occurs for the super-metal-rich models. Although the radiative opacity is still affected by the metallicity increase, the effect on both bolometric luminosity and effective temperature of an increase of $\Delta Z=0.01$ is larger between $Z=$ 0.02 and 0.03 than between $Z=0.04$ and 0.05 , for example, because the opacity increase (at fixed $T$ and $\rho$ ) for a fixed $\Delta Z$ is larger around $Z \sim Z_{\odot}$ than around $Z=0.04$ (because of a larger percentage increase $\Delta Z / Z$ ) as we have verified on the relevant opacity tables. One should also bear in mind an additional effect. For the assumed helium-enrichment ratio, the increase of initial $\mathrm{He}$ associated with the increase of $Z$ has the effect of increasing the model $T_{\text {eff }}$, compared to an increase of $Z$ at constant $Y$ (see e.g. Cassisi \& Salaris 2013). This compensates, at least partially, the $T_{\text {eff }}$ decrease associated with the $Z$ increase.

From the data in Table 1 one notices that, at fixed total mass, the central H-burning lifetime is strongly affected by the metal content, monotonically increasing with increasing metallicity as a consequence of the lower brightness of the stellar structures.

The huge impact of the metallicity on the opacity stratification of the model envelopes is also demonstrated by the $\sim 2.5$ mag decrease of the RGB bump magnitude, when $Z$ increases from $10^{-5}$ to $Z=0.05$, as shown in the inset in Fig. 3 .

It is worth mentioning the trend of the RGB tip luminosity with metallicity. The luminosity increases steadily from $Z=10^{-5}$ to $Z \approx 0.03$, followed by a slight decrease when the initial metal content increases further. On the other hand, the mass of the He core $\left(M_{\mathrm{cHe}}\right)$ at the He flash decreases monotonically with increasing $Z$ (see Table 1).

One important prediction of stellar model calculations is the transition mass $M_{\mathrm{HeF}}$ between stars that ignite $\mathrm{He}$ in an electron degenerate core, and stars that enter the central He burning phase without experiencing core electron degeneracy (see e.g. Sweigart et al. 1989, 1990; Cassisi \& Castellani 1993). Core mass and bolometric luminosity at He ignition change remarkably over a range of only a few tenths of solar mass, as shown in Fig. 4 for the case $Z=10^{-5}$, for models with and without MS convective core overshooting. For this reason, when discussing the evolution of stars with masses around $M_{\mathrm{HeF}}$, one often speaks of a red giant branch transition. 

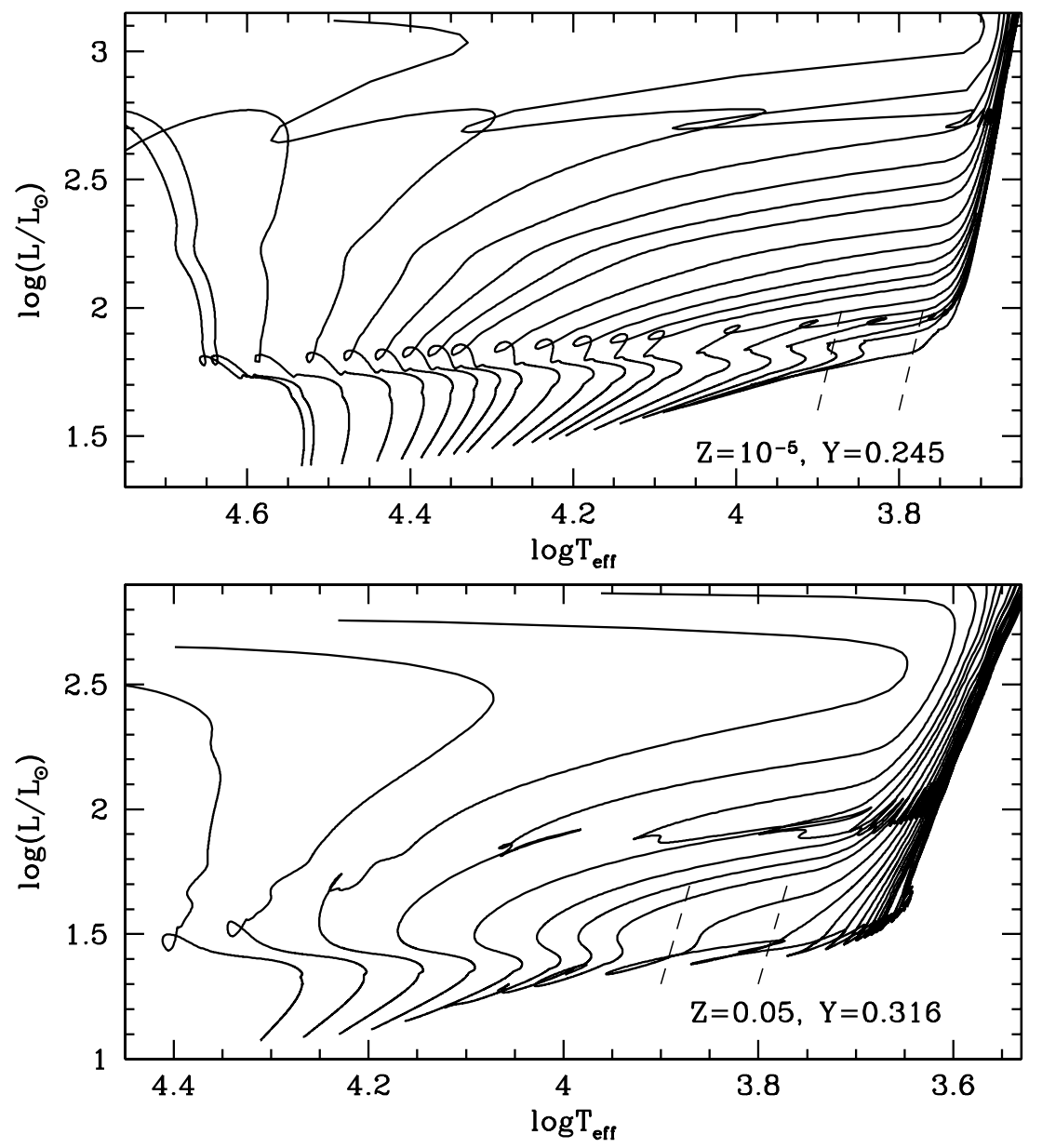

Fig. 2. Upper panel: evolutionary tracks for selected HB models with a $0.8 M_{\odot}$ RGB progenitor with scaled solar $Z=10^{-5}, Y=0.245$. From left to right the tracks are for masses $M / M_{\odot} 0.517,0.518$, and 0.520 , then in steps of $0.005 M_{\odot}$ up to $0.55 M_{\odot}$, in steps of $0.01 M_{\odot}$ up to $0.6 M_{\odot}$, in steps of $0.02 M_{\odot}$ up to $0.7 M_{\odot}$, and then $\Delta M=0.1 M_{\odot}$ up to $0.8 M_{\odot}$. The approximate positions of the blue and red boundaries of the RR Lyrae instability strip are also displayed as thin dashed lines. Lower panel: as in the upper panel, but for $Z=0.05$, $Y=0.316$, and a RGB progenitor with mass equal to $1 M_{\odot}$. The less massive HB model has a mass equal to $0.465 M_{\odot}$. The mass steps are as in the upper panel, but for masses larger than $0.6 M_{\odot}$, where $\Delta M=0.1 M_{\odot}$.

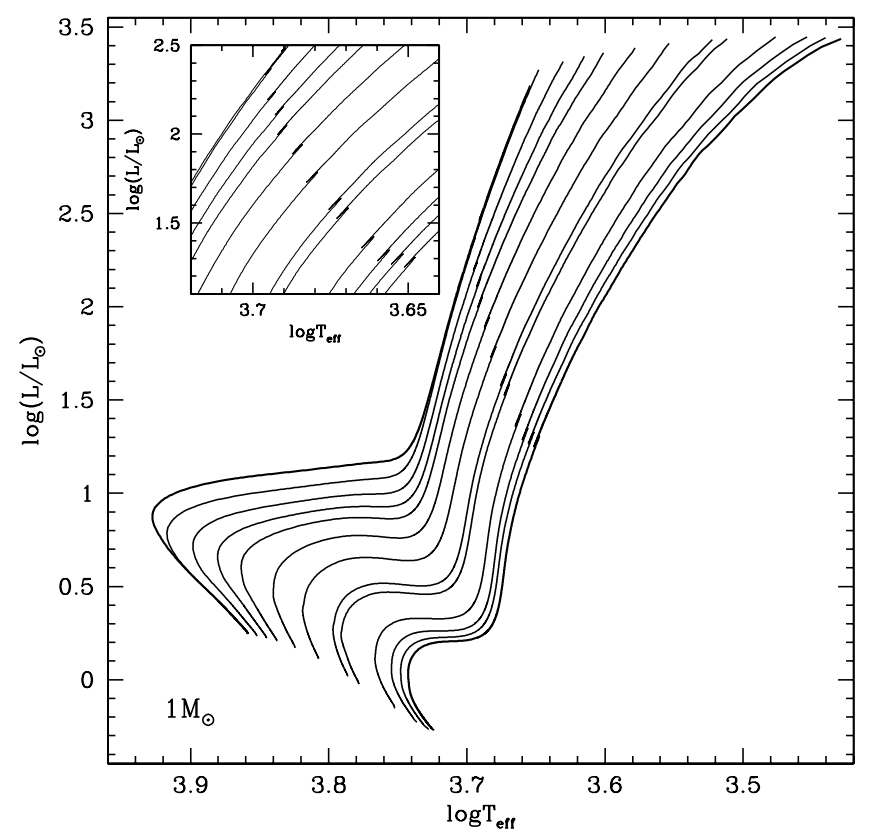

Fig. 3. HR diagrams of $1 M_{\odot}$ models for the full set of BaSTI scaled solar metallicities (from left to right) $Z=10^{-5}, 10^{-4}, 3 \times 10^{-4}, 6 \times 10^{-4}$, $0.001,0.002,0.004,0.008,0.01,0.0198,0.03,0.04$, and 0.05 . The inset shows a zoom on the portion of the RGB bump region.

The value of $M_{\mathrm{HeF}}$ depends on the initial chemical composition, as shown in Fig. 5. In general, an increase of $Z$ at constant $Y$ would cause a monotonic increase of $M_{\mathrm{HeF}}$ as a consequence of the lower MS luminosity and smaller convective cores (at fixed total mass) during the MS. However, the increase of $Y$ with $Z$ due to the adopted $\Delta Y / \Delta Z \sim 1.4$ ratio, favours the thermal conditions required to ignite He burning, hence a decrease of $M_{\mathrm{HeF}}$. This helps to explain the almost constant value of this parameter in the metallicity range from $Z \approx 3 \times 10^{-4}$ to $\sim 10^{-2}$. At higher $Z$, up to $Z \sim 0.03$ the effect associated with the metallicity increase dominates, whilst for $Z>0.03$, the significant $\mathrm{He}$ increase forces $M_{\mathrm{HeF}}$ to decrease.

It is well known that the value of $M_{\mathrm{HeF}}$, for a given chemical composition, depends on the assumed efficiency of the core convective overshooting during the central H-burning stage: the larger the overshooting region, the smaller is $M_{\mathrm{HeF}}$ (see Fig. 5). This is due to the larger He core mass at the end of the central H-burning stage of the models with MS core overshooting; a larger He core mass at the start of the RGB stage implies a hotter core thermal stratification, which favours the thermal conditions required for He-burning ignition. It is worth noticing how the effect of MS convective core overshooting vanishes at the lowest metallicity. This is due to the huge decrease of the size of the convective core during the central H-burning stage in very metalpoor stars, caused by the very low efficiency of the CNO cycle compared to the p-p chain.

As already mentioned, Fig. 2 displays the evolutionary tracks of selected models along both core and shell He-burning stages for the new chemical compositions presented in this paper. They originate from a $\sim 13$ Gyr old RGB progenitor, with an initial mass $M=0.8 M_{\odot}$ for $Z=10^{-5}$, and $1 M_{\odot}$ for $Z=0.05$. Super-metal-rich HB stars have a cooler zero age horizontal branch (ZAHB) location compared to the extremely metal-poor 
Table 1. Selected evolutionary properties of a $1 M_{\odot}$ stellar model for all BaSTI metallicities (scaled solar mixture).

\begin{tabular}{lccccccccc}
\hline \hline$Z$ & $Y$ & $t_{\mathrm{H}}(\mathrm{Gyr})$ & $\log \left(L_{\mathrm{Bump}} / L_{\odot}\right)$ & $\log \left(L_{\text {tip }} / L_{\odot}\right)$ & $M_{\mathrm{cHe}}\left(M_{\odot}\right)$ & $\Delta Y_{\text {surf }}$ & $t_{\text {tip }}(\mathrm{Gyr})$ & $M_{\mathrm{ZAHB}}^{3.85}\left(M_{\odot}\right)$ & $\log \left(L_{\mathrm{ZAHB}}^{3.85} / L_{\odot}\right)$ \\
\hline $10^{-5}$ & 0.245 & 5.35 & 2.477 & 3.183 & 0.5103 & 0.013 & 5.61 & -- & -- \\
$10^{-4}$ & 0.245 & 5.25 & 2.352 & 3.271 & 0.4972 & 0.016 & 5.57 & 0.801 & 1.757 \\
$3 \times 10^{-4}$ & 0.245 & 5.31 & 2.217 & 3.315 & 0.4924 & 0.018 & 5.69 & 0.707 & 1.710 \\
$6 \times 10^{-4}$ & 0.246 & 5.41 & 2.132 & 3.342 & 0.4895 & 0.019 & 5.84 & 0.665 & 1.685 \\
$10^{-3}$ & 0.246 & 5.58 & 2.024 & 3.361 & 0.4876 & 0.020 & 6.07 & 0.640 & 1.664 \\
$2 \times 10^{-3}$ & 0.248 & 5.96 & 1.915 & 3.387 & 0.4853 & 0.020 & 6.55 & 0.609 & 1.631 \\
$4 \times 10^{-3}$ & 0.251 & 6.72 & 1.757 & 3.412 & 0.4829 & 0.021 & 7.48 & 0.585 & 1.593 \\
$8 \times 10^{-3}$ & 0.256 & 8.09 & 1.608 & 3.433 & 0.4798 & 0.023 & 9.10 & 0.564 & 1.543 \\
$10^{-2}$ & 0.259 & 8.75 & 1.555 & 3.437 & 0.4785 & 0.022 & 9.84 & 0.558 & 1.521 \\
0.0198 & 0.2734 & 11.29 & 1.391 & 3.446 & 0.4727 & 0.023 & 12.61 & 0.538 & 1.461 \\
0.03 & 0.288 & 12.54 & 1.318 & 3.447 & 0.4675 & 0.023 & 13.94 & 0.526 & 1.431 \\
0.04 & 0.303 & 13.07 & 1.297 & 3.441 & 0.4621 & 0.021 & 14.49 & 0.516 & 1.419 \\
0.05 & 0.316 & 13.18 & 1.279 & 3.436 & 0.4574 & 0.021 & 14.55 & 0.508 & 1.417 \\
\hline
\end{tabular}

Notes. $t_{\mathrm{H}}$ and $t_{T i p}$ denote the age of the model at central $\mathrm{H}$ exhaustion and at the tip of the RGB, respectively. $\Delta Y_{\text {surf }}$ is the amount of extra helium dredged to the surface during the first dredge-up. $M_{\mathrm{ZAHB}}^{3.85}$ and $\log \left(L_{\mathrm{ZAHB}}^{3.85} / L_{\odot}\right)$ are the total mass and surface luminosity of the BaSTI HB models whose ZAHB locations are at $\log T_{\text {eff }}=3.85$, taken as representative of the average effective temperature of the RR Lyrae instability strip.

Table 2. Selected evolutionary properties for $4 M_{\odot}$ models computed with and without MS convective core overshooting for all scaled-solar BaSTI metallicities.

\begin{tabular}{lcccccccc}
\hline \hline$Z$ & $Y$ & $M_{\mathrm{cc}}\left(M_{\odot}\right)$ & $t_{\mathrm{H}}(\mathrm{Myr})$ & $M_{\mathrm{cHe}}\left(M_{\odot}\right)$ & $\log T_{\mathrm{eff}}$ & $t_{\mathrm{He}}(\mathrm{Myr})$ & $\Delta Y_{\text {surf }}$ & $M_{\mathrm{cCO}}\left(M_{\odot}\right)$ \\
\hline \multicolumn{7}{c}{ No MS core overshooting } \\
\hline $10^{-5}$ & 0.245 & 0.9769 & 101.41 & 0.5256 & 4.154 & 24.42 & 0.062 & 0.8225 \\
$10^{-4}$ & 0.245 & 1.0255 & 106.64 & 0.5282 & 4.038 & 24.08 & 0.059 & 0.8248 \\
$3 \times 10^{-4}$ & 0.245 & 1.0356 & 109.21 & 0.5282 & 3.937 & 24.80 & 0.056 & 0.8234 \\
$6 \times 10^{-4}$ & 0.246 & 1.0376 & 114.10 & 0.5232 & 3.817 & 26.31 & 0.050 & 0.8209 \\
$10^{-3}$ & 0.246 & 1.0405 & 112.62 & 0.5193 & 3.731 & 28.26 & 0.048 & 0.8190 \\
$2 \times 10^{-3}$ & 0.248 & 1.0505 & 116.06 & 0.5123 & 3.699 & 31.29 & 0.036 & 0.8131 \\
$4 \times 10^{-3}$ & 0.251 & 1.0117 & 119.71 & 0.4993 & 3.686 & 34.65 & 0.022 & 0.7978 \\
$8 \times 10^{-3}$ & 0.256 & 0.9751 & 126.35 & 0.4891 & 3.672 & 37.54 & 0.008 & 0.7846 \\
$10^{-2}$ & 0.259 & 0.9751 & 129.61 & 0.4854 & 3.667 & 38.75 & 0.005 & 0.7780 \\
0.0198 & 0.2734 & 0.9221 & 140.34 & 0.4812 & 3.650 & 45.87 & no $2^{\text {nd }} \mathrm{DU}$ & 0.7143 \\
0.03 & 0.288 & 0.8850 & 144.58 & 0.4851 & 3.640 & 49.11 & no $2^{\text {nd }} \mathrm{DU}$ & 0.6840 \\
0.04 & 0.303 & 0.8459 & 143.61 & 0.4930 & 3.633 & 49.12 & no $2^{\text {nd }} \mathrm{DU}$ & 0.6823 \\
0.05 & 0.316 & 0.8374 & 142.80 & 0.5052 & 3.627 & 46.52 & no $2^{\text {nd }} \mathrm{DU}$ & 0.6971 \\
\hline \multicolumn{7}{c}{} & & \multicolumn{7}{c}{$\mathrm{MS}$ core overshooting } & & & \\
\hline $10^{-5}$ & 0.245 & 0.9808 & 124.25 & 0.6208 & 4.053 & 15.42 & 0.085 & 0.8477 \\
$10^{-4}$ & 0.245 & 1.0367 & 129.68 & 0.6329 & 3.843 & 15.54 & 0.087 & 0.8557 \\
$3 \times 10^{-4}$ & 0.245 & 1.0391 & 132.13 & 0.6315 & 3.712 & 16.21 & 0.085 & 0.8516 \\
$6 \times 10^{-4}$ & 0.246 & 1.0406 & 133.81 & 0.6272 & 3.696 & 16.80 & 0.082 & 0.8510 \\
$10^{-3}$ & 0.246 & 1.0427 & 135.80 & 0.6219 & 3.689 & 17.49 & 0.079 & 0.8465 \\
$2 \times 10^{-3}$ & 0.248 & 1.0265 & 142.76 & 0.6114 & 3.678 & 18.02 & 0.069 & 0.8365 \\
$4 \times 10^{-3}$ & 0.251 & 1.0056 & 145.64 & 0.5959 & 3.665 & 19.06 & 0.059 & 0.8290 \\
$8 \times 10^{-3}$ & 0.256 & 0.9672 & 152.68 & 0.5814 & 3.651 & 21.18 & 0.054 & 0.8108 \\
$10^{-2}$ & 0.259 & 0.9566 & 158.80 & 0.5774 & 3.645 & 23.51 & 0.052 & 0.8014 \\
0.0198 & 0.2734 & 0.9082 & 171.17 & 0.5673 & 3.629 & 28.93 & 0.041 & 0.7787 \\
0.03 & 0.288 & 0.8781 & 175.48 & 0.5703 & 3.618 & 31.25 & 0.037 & 0.7696 \\
0.04 & 0.303 & 0.8583 & 174.45 & 0.5796 & 3.611 & 29.64 & 0.035 & 0.7659 \\
0.05 & 0.316 & 0.8487 & 170.05 & 0.5903 & 3.605 & 28.85 & 0.035 & 0.7655 \\
\hline
\end{tabular}

Notes. $M_{\mathrm{cc}}$ is the convective core mass at the beginning of central H-burning; $t_{\mathrm{H}}$ corresponds to the age at the central $\mathrm{H}$ exhaustion; $M_{\mathrm{cHe}}\left(M_{\odot}\right)$ and $\log T_{\text {eff }}$ are the helium core mass and the logarithm of the effective temperature at the ignition of central He-burning, respectively; $t_{\mathrm{He}}$ is the core helium burning lifetime; $\Delta Y_{\text {surf }}$ is the amount of extra helium dredged to the surface during the second dredge-up; $M_{\mathrm{cCO}}\left(M_{\odot}\right)$ is the C-O core mass at the first thermal pulse.

counterpart with the same total mass because of the higher envelope opacity and smaller He core. Metal-rich HB models need to experience a huge mass loss along the RGB to be able to reach ZAHB effective temperatures hotter than $\sim 10000 \mathrm{~K}$; for instance for $Z=0.05$, the stellar model whose ZAHB location is at $T_{\text {eff }}=10000 \mathrm{~K}$ has a total mass equal to $\approx 0.50 M_{\odot}$, corresponding to an amount of mass lost by the RGB progenitor equal to $\Delta M \approx 0.5 M_{\odot}$, i.e. about $50 \%$ of the initial mass. On the other hand, all the very metal-poor models, regardless of the amount of mass lost during the RGB, have a ZAHB location hotter than 


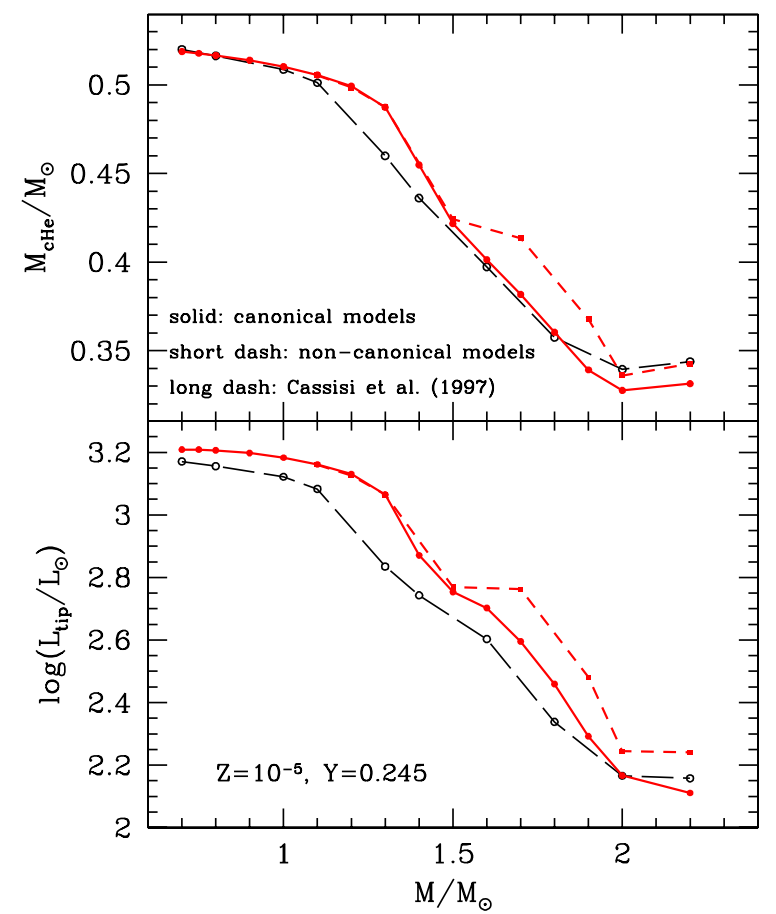

Fig. 4. Upper panel: behaviour of $M_{\mathrm{cHe}}$ at the RGB tip as a function of the initial mass for the scaled-solar $Z=10^{-5}$ chemical composition, and for both canonical (no convective core overshooting along the MS) and non-canonical (convective core overshooting along the MS) stellar models. Solid and short dashed lines correspond to the present calculations, the long dashed line to the results by Cassisi et al. (1997). Lower panel: as in the upper panel, but for the trend of the stellar surface luminosity with mass at the RGB tip.

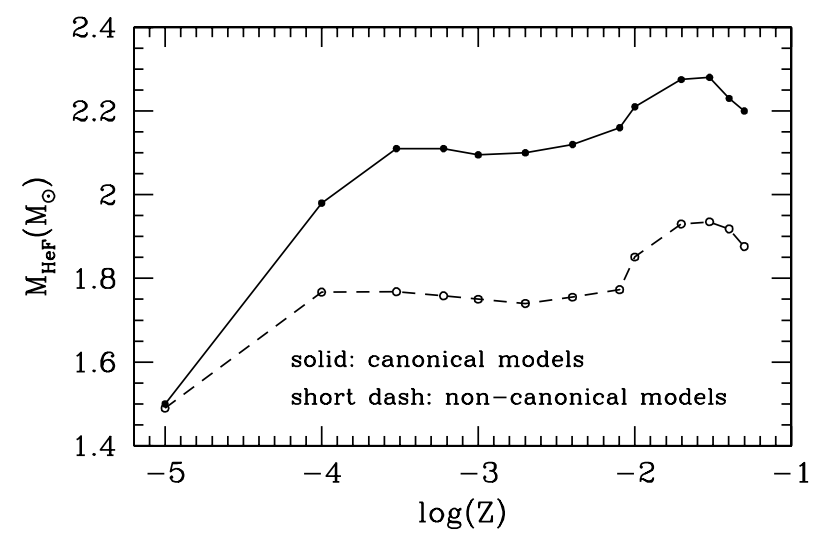

Fig. 5. Behaviour of $M_{\mathrm{HeF}}$ with metallicity as predicted by BaSTI models for the scaled-solar metal mixture, both with (non-canonical models) and without (canonical models) MS convective core overshooting.

the blue boundary of the RR Lyrae instability strip. This implies that both extremely metal-poor and super-metal-rich stellar populations have a very small, perhaps non-existent, probability to produce significant populations of RR Lyrae variable stars (see also Cassisi et al. 1997 and Bono et al. 1997).

Figure 6 shows the ZAHBs for all BaSTI scaled solar compositions. They obviously become progressively fainter and cooler with increasing metallicity, because of the previously mentioned larger envelope opacities, but mainly because of the smaller He core at He ignition. It is worth noticing the behaviour of the $Z=0.05 \mathrm{ZAHB}$, whose brightness at $T_{\text {eff }}<10000 \mathrm{~K}$ coincides with that of the $Z=0.04$ counterpart. This can be

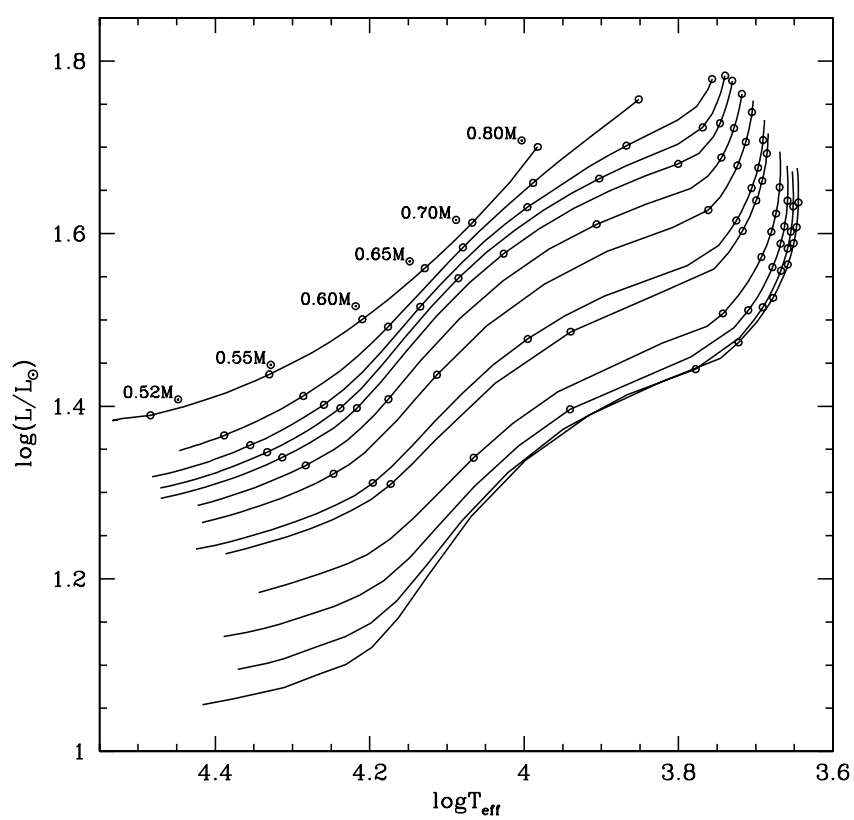

Fig. 6. HR diagram of ZAHBs for the complete set of scaled solar metallicities ( $Z$ increases from top to bottom). Filled circles correspond to selected models with $M / M_{\odot}=0.52,0.55,0.60,0.65,0.70$, and 0.80 .

explained as follows. The ZAHB brightness depends on the mass of the He core and on the shell H-burning efficiency which is controlled by both the mass and chemical composition of the envelope. At the hot end of the ZAHB the envelope mass is tiny, the $\mathrm{H}$-burning efficiency is vanishing, and therefore the ZAHB luminosity is only a function of the He core mass. Given that $M_{\mathrm{cHe}}$ is smaller at $Z=0.05$ compared to $Z=0.04$, the ZAHB models are correspondingly fainter. When the envelope mass increases (that moves the ZAHB location towards the cool side of the HR diagram) shell burning provides a major contribution to the energy budget. Given that $Z=0.05$ models have a larger envelope He and CNO abundances (see data in Table 1), the shell burning is more efficient and compensates for the smaller He core mass. As a consequence, the ZAHB for $Z=0.04$ and $Y=0.303$ overlaps the ZAHB for $Z=0.05$ and $Y=0.316$ below a given $T_{\text {eff }}$.

Some relevant evolutionary and structural properties of $4 M_{\odot}$ models, representative of intermediate-mass stars, computed with and without MS convective core overshooting, for all scaled solar metallicities of the BaSTI archive are listed in Table 2. A comparison between canonical (no overshooting) and noncanonical (with overshooting) evolutionary tracks of a $4 M_{\odot}$ stellar model, for $Z=0.00001$ and 0.05 , is shown in Fig. 7 .

It is important to notice that the strong dependence of the radiative opacity on metals affect the properties of the intermediate-mass models in the same way as for the low-mass ones. Tracks shown in Fig. 1 reveal that at $Z=10^{-5}$ the transition from lower (H-burning occurring mainly via the $\mathrm{p}-\mathrm{p}$ chain) to upper (dominated by the CNO cycle) MS stars happens at $\sim 2.3 M_{\odot}$, as testified by the appearance of the overall contraction phase at central $\mathrm{H}$ exhaustion. For $Z=0.05$ the transition occurs at $\sim 1.1 M_{\odot}$. This huge difference is present because when the metallicity decrease, the abundance of CNO elements also decreases, and much higher central temperatures (hence larger masses) are needed for the CNO cycle energy generation to dominate over the $\mathrm{p}-\mathrm{p}$ chain contribution.

The generally higher central temperatures of the metal-poor models also imply that for intermediate-mass models the thermal conditions for He-burning ignition are reached earlier than 
A. Pietrinferni et al.: Extension of the BaSTI model archive

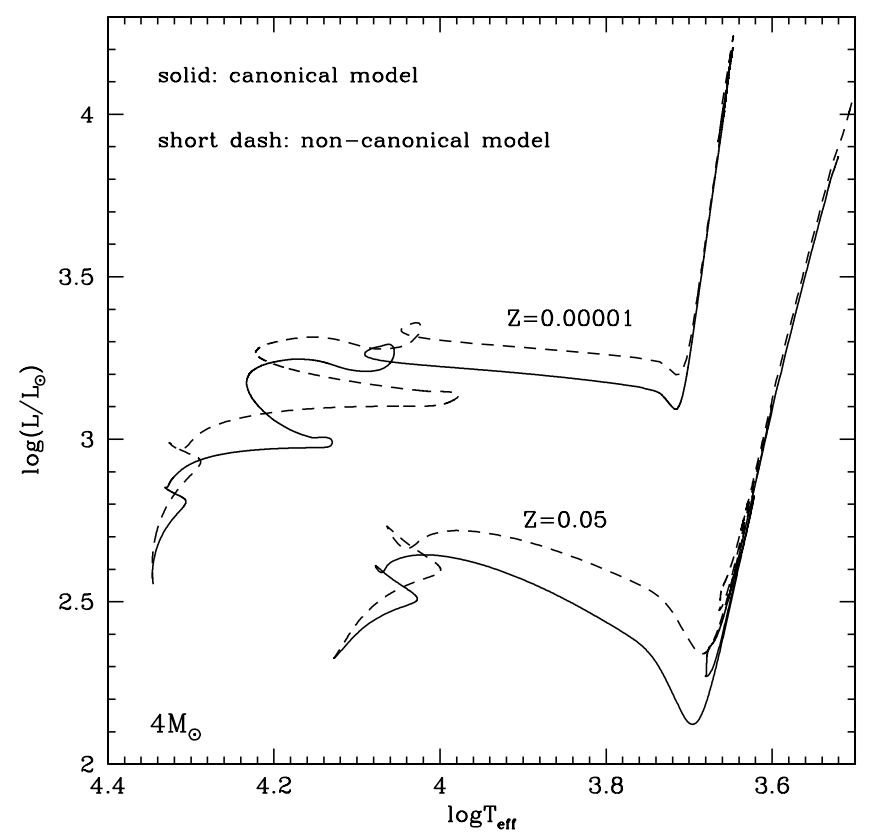

Fig. 7. Evolutionary tracks for $4.0 M_{\odot}$ stellar models computed with (non-canonical) and without (canonical) MS convective core overshooting, for both $Z=10^{-5}$ and 0.05 .

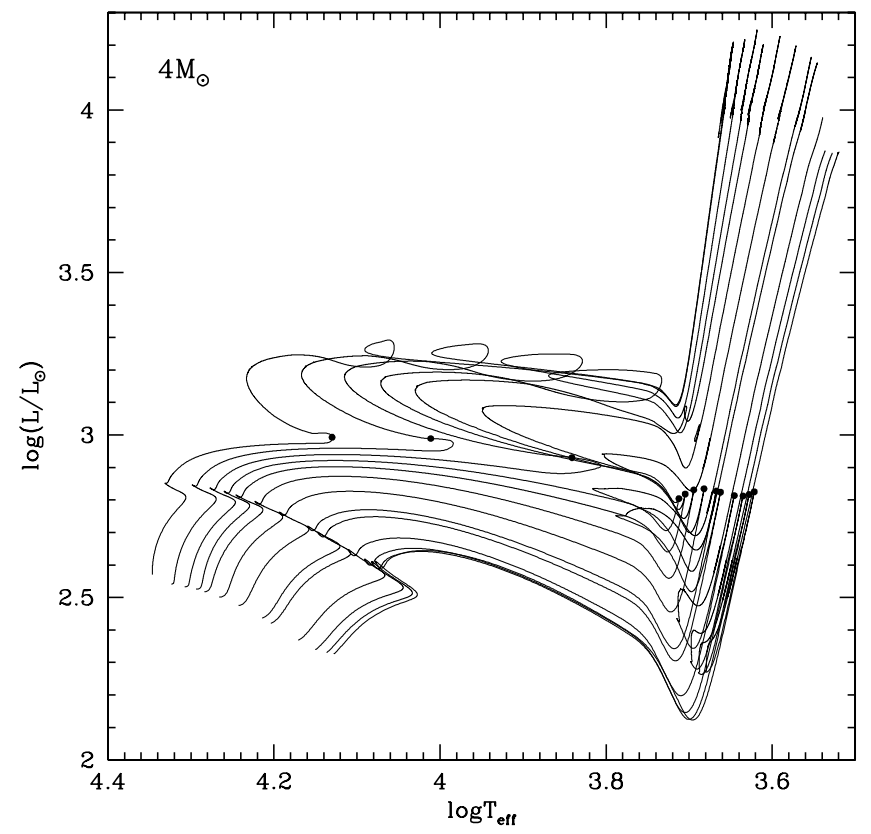

Fig. 8. Evolutionary tracks for $4.0 M_{\odot}$ models (no overshooting) and all scaled solar chemical compositions of the BaSTI database. Filled circles mark central He-burning ignition.

for their metal-rich counterparts. Therefore, at very low $Z$, intermediate-mass models miss the RGB stage, as shown in Fig. 8.

\section{Comparison with observations and literature models}

There are not many observations that sample the two extreme metallicities discussed in this paper, and the available ones are not accurate enough to enable stringent tests of the models.

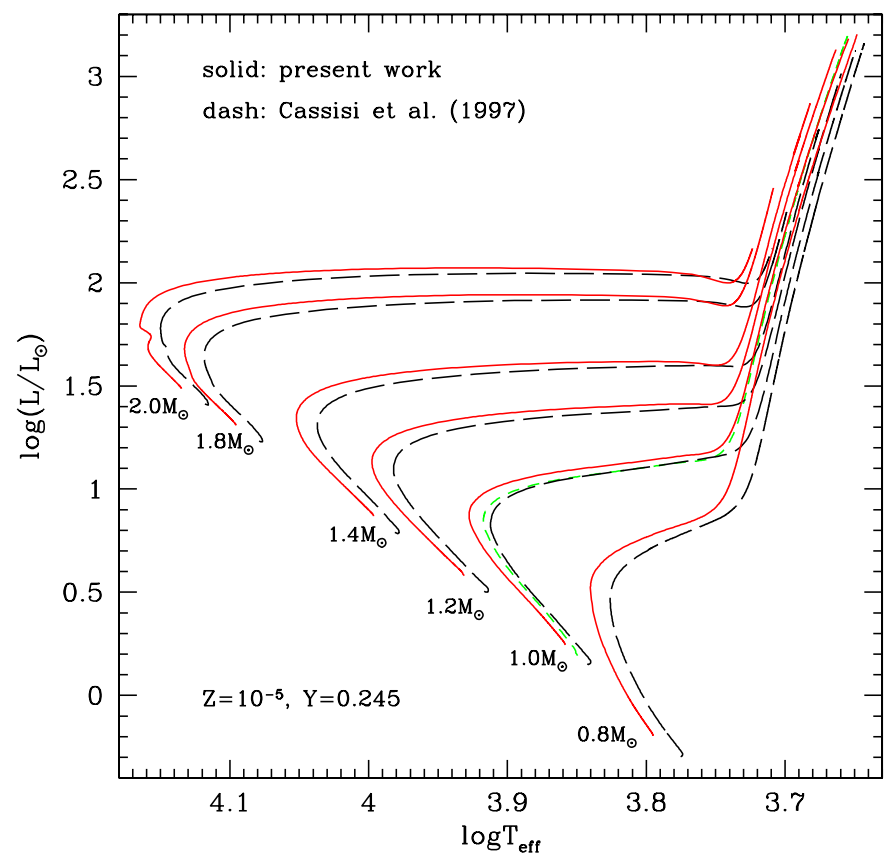

Fig. 9. HR diagram of selected H-burning models compared to Cassisi et al. (1997) results. The short dashed line corresponds to a $1 M_{\odot}$ model computed with our updated physical inputs but with the same initial He content adopted by Cassisi et al. (1997).

We show in Fig. 10 a comparison of the CMD of selected RGB stars in the metal-poor Boötes I dwarf spheroidal galaxy (Norris et al. 2008), with 12.5 Gyr $\alpha$-enhanced theoretical isochrones and various $[\mathrm{Fe} / \mathrm{H}]$ values (the precise value of the age is not critical). The observational points have $[\mathrm{Fe} / \mathrm{H}]$ determinations in the range between $[\mathrm{Fe} / \mathrm{H}] \sim-1.5$ and $[\mathrm{Fe} / \mathrm{H}] \sim-3.5$, with standard deviations on the individual measurements between $\sim 0.19$ and $\sim 0.35$ dex. A distance modulus $(m-M)_{0}=18.96$ from Kuehn et al. (2008) has been applied to the isochrones. Observational data were dereddened by Norris et al. (2008) using $E(B-V)=0.02$. Taking into account the substantial error in the $[\mathrm{Fe} / \mathrm{H}]$ estimates, there is a broad agreement between models and observations.

Figure 11 compares the $\log (g)-T_{\text {eff }}$ diagram of a $12.5 \mathrm{Gyr}$, $[\mathrm{Fe} / \mathrm{H}]-3.62\left(Z=10^{-5}\right) \alpha$-enhanced isochrone, with the extremely metal-poor star Boo-1137, in the same dwarf spheroidal galaxy. This star has a spectroscopic determination of $[\mathrm{Fe} / \mathrm{H}]=$ $-3.7 \pm 0.1$ (and $\alpha$-element enhancement; Norris et al. 2010). Error bars quoted by the authors are also displayed. Within the error bars, the extremely metal-poor models discussed in this paper are able to match the observations.

Stellar models for these extreme metallicities have been calculated, so far, by few authors. Models for $Z=10^{-5}$, covering both low- and intermediate-mass stars have been computed by Cassisi et al. (1997), and can be also found as part of the YaleYonsei (YY) model database (see e.g. Demarque et al. 2004). Figure 4 shows the comparison between our models (without convective overshooting, as in the Cassisi et al. 1997 calculations) and Cassisi et al. (1997) results, concerning both the He core mass and surface luminosity at the RGB tip, for stellar masses around the RGB transition, while Fig. 9 compares the HR diagrams of selected H-burning models.

The differences of the evolutionary tracks are due to the the completely different physics inputs adopted, but we also need to consider that the Cassisi et al. (1997) computations assume an initial $Y=0.23$, while we now employ an updated and higher 


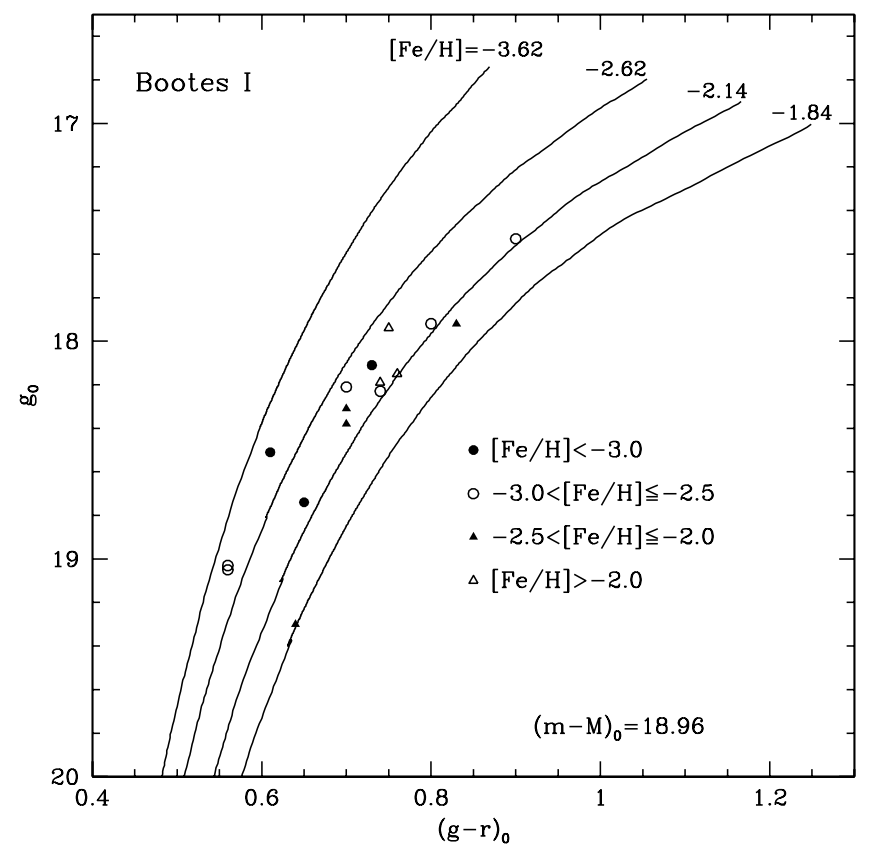

Fig. 10. Colour-magnitude diagram of a sample of metal-poor RGB stars belonging to the Boötes I dwarf spheroidal galaxy that have spectroscopic $[\mathrm{Fe} / \mathrm{H}]$ determinations (Norris et al. 2008). Theoretical isochrones for an age of $12.5 \mathrm{Gyr}$ and the labelled $[\mathrm{Fe} / \mathrm{H}]$ values (for an $\alpha$-enhanced metal mixture) are also displayed (see text for details).

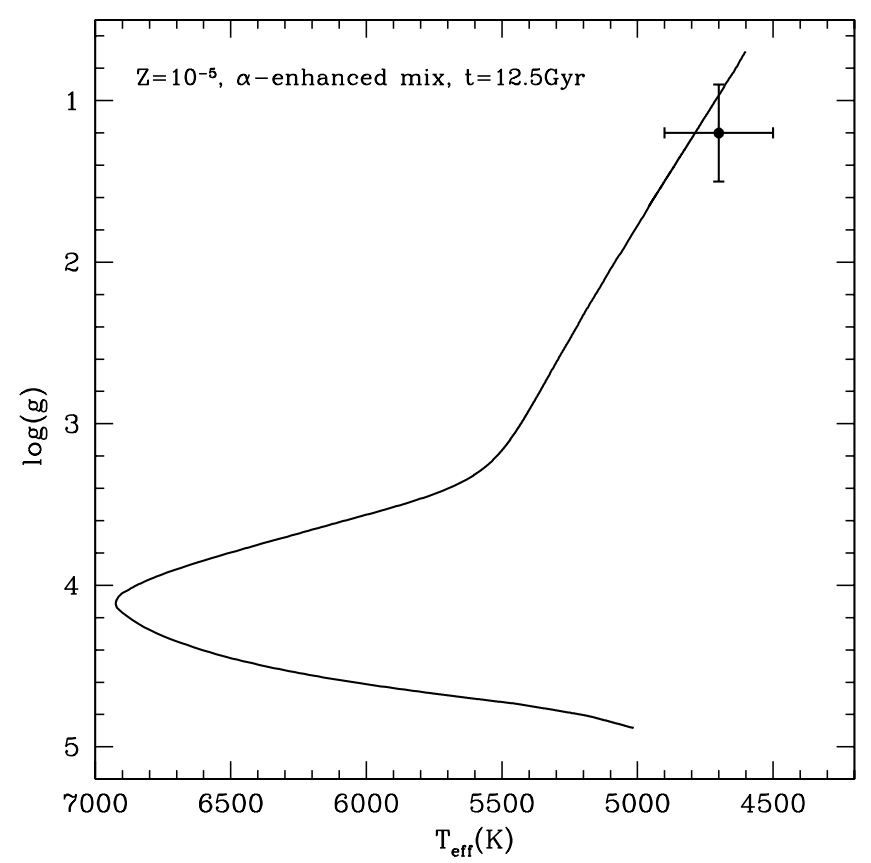

Fig. 11. Comparison of a $12.5 \mathrm{Gyr},[\mathrm{Fe} / \mathrm{H}]=-3.62 \alpha$-enhanced isochrone in the $\log (g) T_{\text {eff }}$ diagram, with the extremely metal-poor $([\mathrm{Fe} / \mathrm{H}]=-3.7 \pm 0.1)$ star Boo-1137, in the Boötes I dwarf spheroidal galaxy (Norris et al. 2010).

estimate of the primordial He abundance $(Y=0.245)$. To isolate this effect, we performed an additional calculation for a $1 M_{\odot}$ model by employing the BaSTI physics inputs, but with $Y=0.23$, as in Cassisi et al. (1997). This evolutionary track is shown in Fig. 9 to be very close to the old computations along the MS, while matching the new BaSTI results along the RGB. This implies that along the RGB the difference with the Cassisi et al. (1997) models is due to the different low temperature
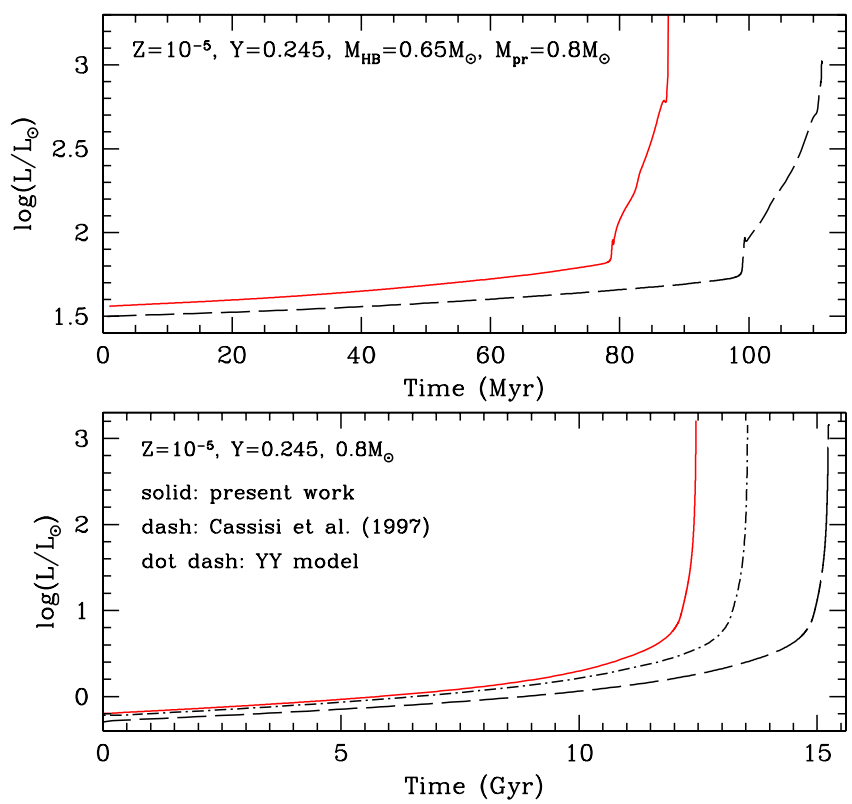

Fig. 12. Upper panel: behaviour of the surface luminosity as a function of time for two HB models with the same mass (see labels). The solid line corresponds to our computations, while the dashed line displays results by Cassisi et al. (1997). Lower panel: as in the upper panel, but for $0.8 M_{\odot}$ calculations from the zero age MS to the RGB tip, also including a YY model.

radiative opacities, while it is the different initial He abundance that mainly causes the differences along the MS and subgiant branch.

As for the evolutionary lifetimes, the lower panel of Fig. 12 compares the $0.8 M_{\odot}$ luminosity-age relation with the Cassisi et al. (1997) counterpart. The MS lifetime is shorter by about 2.5 Gyr in our computations, a consequence of both the larger initial He content and the different physics inputs (a fundamental role is played by the different equation of state). The upper panel of the same figure compares the trend of the luminosity with time of a HB model originated from a $0.8 M_{\odot}$ RGB progenitor, with the Cassisi et al. (1997) counterpart. Our model has a core He-burning lifetime that is about $20 \%$ shorter than the older calculations. This result is a consequence of different physics inputs, in particular the use of an updated nuclear cross section for the ${ }^{12} \mathrm{C}(\alpha, \gamma){ }^{16} \mathrm{O}$ reaction (Kunz et al. 2002).

Figure 13 shows HR diagrams of selected HB models. There is a relatively small luminosity difference that is mainly a consequence of the different envelope He abundance, for the RGB progenitors have a similar He core mass at the RGB tip (see data in Fig. 4).

Figure 14 displays a comparison similar to Fig. 9, but with YY models. Here we consider BaSTI models with convective core overshooting, because it is included (in a similar way as BaSTI) in the YY calculations. This set adopts physics inputs that are very similar to our calculations, the main differences being the inclusion of He diffusion (that affects the lower mass models) and a lower initial He abundance, $Y=0.23$. For masses above $\sim 1.2 M_{\odot}$ the differences in the HR diagrams of the two sets of tracks are due essentially to the different initial $Y$, while for the lower masses the effect of He diffusion amplifies the differences around the turn off (TO). The RGBs of the two sets of calculations are almost identical, for they are very weakly affected by the initial $Y$ and efficiency of atomic diffusion. Evolutionary lifetimes during the core and shell H-burning 
A. Pietrinferni et al.: Extension of the BaSTI model archive

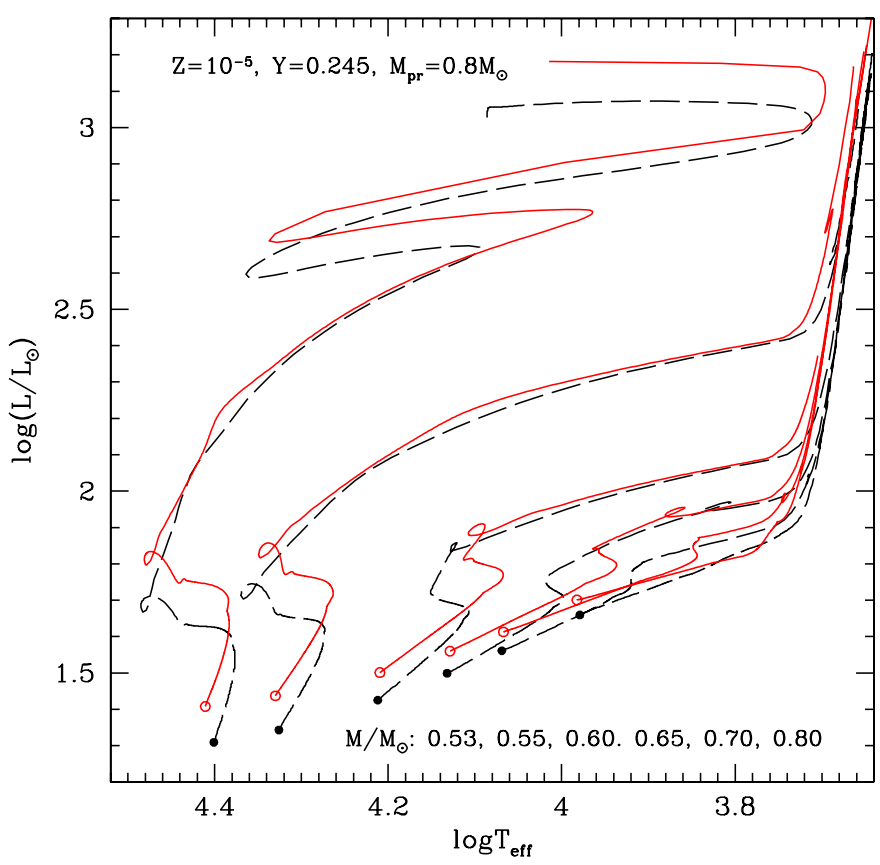

Fig. 13. As in Fig. 12, but for the HR diagram of selected HB models with the same RGB progenitor mass $\left(M_{\mathrm{pr}}\right)$.

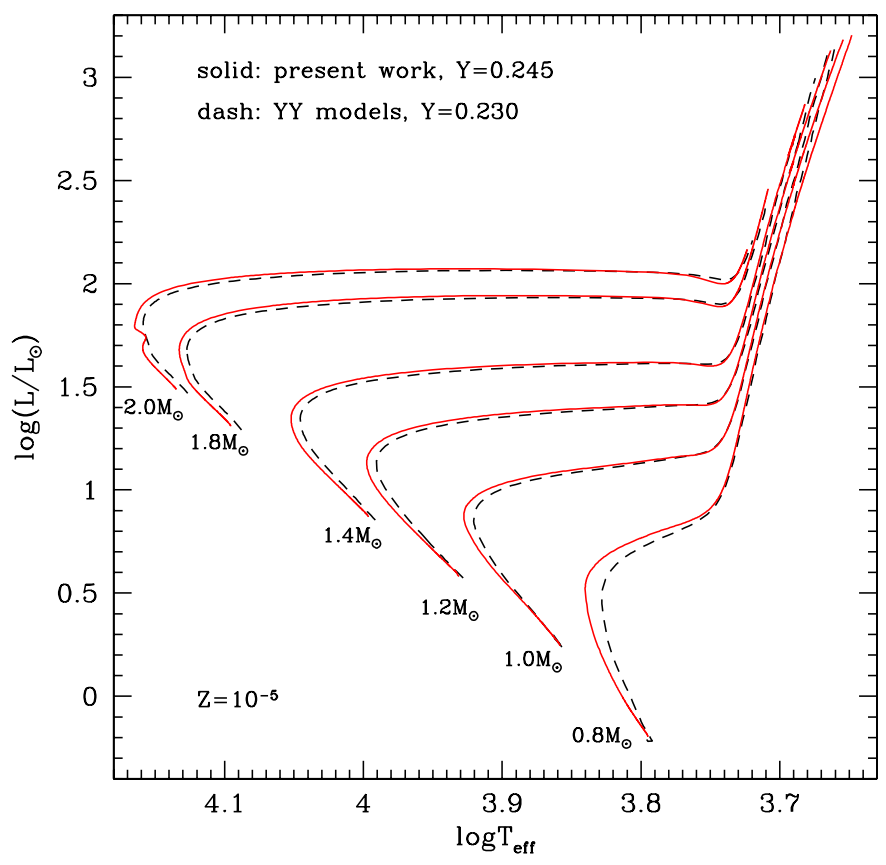

Fig. 14. As in Fig. 9, but for a comparison with YY models.

stages for the $0.8 M_{\odot}$ YY model are displayed in the lower panel of Fig. 12; they turn out to be longer by $\sim 1$ Gyr for the MS compared to BaSTI models, in spite of the efficient atomic diffusion that tends to shorten the MS lifetime compared to the nodiffusion case. The main reason for this difference is very likely the higher initial He abundance in the BaSTI models that, as mentioned already, decreases the MS lifetime.

The only possibility we have to compare the $Z=0.05$ models with independent calculations at exactly the same $Z$ is to consider the models by Bressan et al. (2012, PARSEC models). The PARSEC library includes a grid point at $Z=0.05$, although with a larger initial He content. The physics inputs of PARSEC calculations are similar to those adopted for the BaSTI

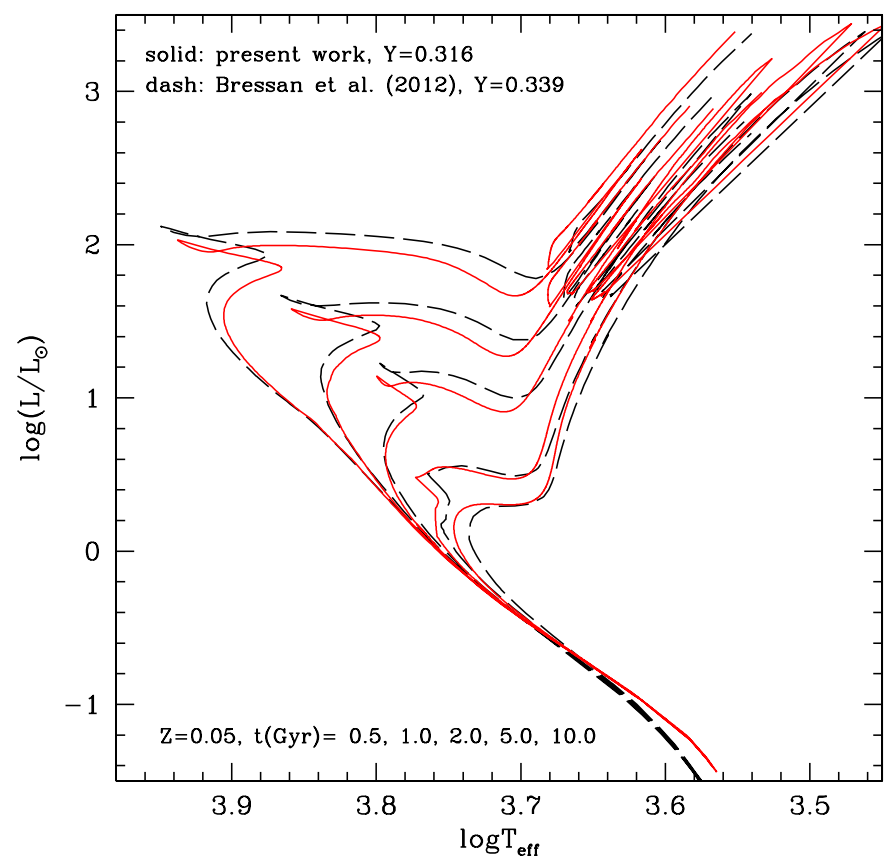

Fig. 15. Selected isochrones from BaSTI and the PARSEC databases for $Z=0.05$. The BaSTI isochrones include convective core overshooting during the central H-burning stage.

database, the main differences being the low- $T$ and electron conduction opacities, and some nuclear reaction rates, plus their inclusion of atomic diffusion. Their adopted scaled solar heavy element mixture starts from Grevesse \& Sauval (1998), but supplemented for a subset of elements by the Caffau et al. (2011, and references therein) results. In particular, the very abundant $\mathrm{CNO}$ elements and $\mathrm{Fe}$ are amongst the metals with Caffau et al. (2011) abundances, and the resulting metal mixture is appreciably different from our calculations. Figure 15 displays a comparison of selected isochrones from BaSTI and PARSEC, from the MS to the asymptotic giant branch phase. We display the BaSTI predictions accounting for core convective overshoot, because the PARSEC calculations account for this non-canonical mixing process.

The two sets of isochrones display differences along the various branches that are not very large but are still noticeable. For isochrones populated by stars with well-developed convective cores along the MS (ages below 5 Gyr), PARSEC calculations display typically brighter and hotter TOs, while the reverse is true for the older ages. The RGB and asymptotic giant branch $T_{\text {eff }}$ of our models is typically larger (the reverse is true for the lower MS), and the central He burning luminosity is generally fainter. It is difficult to disentangle the various causes of these differences that are undoubtedly due to the different initial $\mathrm{He}$ (which however, should not appreciably affect the RGB $T_{\text {eff }}$, and would in any case exacerbate the differences along this phase), the efficiency of atomic diffusion (for the older isochrone TO region only), a slightly different extension of the overshooting region (larger in the PARSEC models) in stars with well-developed MS convective cores, and the different initial metal mixture.

\section{Summary}

We have presented an extension of the BaSTI stellar evolution database to extremely metal-poor $\left(Z=10^{-5}\right)$ and supermetal-rich $(Z=0.05)$ metallicities, with both scaled-solar and $\alpha$-enhanced $([\alpha / \mathrm{Fe}]=0.4)$ heavy element distributions. With 
these new additions, the BaSTI database can be used to model stellar populations ranging from the most metal-poor components of faint dwarf galaxies in the Local Group, to the metalrich populations of the Galactic bulge.

We have discussed the variations of several fundamental predictions of stellar evolution over the large metallicity range spanned by the full BaSTI models, and compared the new calculations with literature models at $Z=10^{-5}$ and $Z=0.05$. The comparison discloses a good agreement with $\mathrm{YY}$ calculations from the MS to the tip of the RGB (YY models employ very similar input physics) at $Z=10^{-5}$. The existing small differences in the HR diagram are easily explained by the different initial $\mathrm{He}$ abundance, and the inclusion of He diffusion in YY calculations. The comparison with the Cassisi et al. (1997) $Z=10^{-5}$ models shows that the $T_{\text {eff }}$ difference along the RGB is due to the different low temperature radiative opacities, while it is the different initial He abundance that causes the differences in $T_{\text {eff }}$ and bolometric luminosity along the MS and subgiant branch. The Cassisi et al. (1997) calculations also include HB models, which tend to be slightly fainter mainly because of the lower initial $Y$.

The only existing modern calculations at $Z=0.05$ are from the PARSEC database, and we compared isochrones for selected ages between 0.5 and 10.0 Gyr. Several differences appear between the two sets, whose causes are difficult to disentangle. They are due to the different initial $\mathrm{He}$, the inclusion of atomic diffusion in the PARSEC models (for the older isochrone TO region only), a slightly different extension of the overshooting region in stars with well developed MS convective cores and the different initial metal mixture.

Acknowledgements. We warmly thank our referee for the pertinent comments that have improved the readability of an early version of the manuscript. S.C. is grateful for financial support from PRIN-INAF 2011 "Multiple Populations in Globular Clusters: their role in the Galaxy assembly" (PI: E. Carretta), and from PRIN MIUR 2010-2011, project "The Chemical and Dynamical Evolution of the Milky Way and Local Group Galaxies", prot. 2010LY5N2T (PI: F. Matteucci). This research has made use of the NASA Astrophysics Data System Abstract Service and the SIMBAD database operated at CDS, Strasbourg, France.

\section{References}

Alexander, D. R., \& Ferguson, J. W. 1994, ApJ, 437, 879

Bedin, L. R., Cassisi, S., Castelli, F., et al. 2005, MNRAS, 357, 1038

Bedin, L. R., King, I. R., Anderson, J., et al. 2008, ApJ, 678, 1279

Belokurov, V., Zucker, D. B., Evans, N. W., et al. 2007, ApJ, 654, 897

Belokurov, V., Walker, M. G., Evans, N. W., et al. 2010, ApJ, 712, L103

Böhm-Vitense, E. 1958, Z. Astrophys., 46, 108

Bono, G., Caputo, F., Cassisi, S., Castellani, V., \& Marconi, M. 1997, ApJ, 489, 822
Bressan, A., Marigo, P., Girardi, L., et al. 2012, MNRAS, 427, 127

Brogaard, K., VandenBerg, D. A., Bruntt, H., et al. 2012, A\&A, 543, A106

Burstein, D., Bertola, F., Buson, L. M., Faber, S. M., \& Lauer, T. R. 1988, ApJ, 328,440

Caffau, E., Ludwig, H.-G., Steffen, M., Freytag, B., \& Bonifacio, P. 2011, Sol. Phys., 268, 255

Cassisi, S. 2005, Proc. meeting Resolved Stellar Populations, Cancun, Mexico [arXiv: astro-ph/0506161]

Cassisi, S. 2009, in Proc. meeting Probing stellar populations out to the distant Universe, Cefalú 2008, AIP Conf. Proc., 1111, 55

Cassisi, S. 2010, in Proc. meeting Stellar Populations: Planning for the Next Decade, IAU Symp., 262, 13

Cassisi, S. 2012, in Proc. meeting Red Giants as Probes of the Structure and Evolution of the Milky Way, Astrophys. Space Sci. Proc. (Berlin Heidelberg: Springer-Verlag), 57

Cassisi, S., \& Castellani, V. 1993, ApJS, 88, 509

Cassisi, S., \& Salaris, M. 2013, Old Stellar Populations: how to study the fossil record of galaxy formation (Berlin: Wiley-VCH)

Cassisi, S., Castellani, V., \& Tornambé, A. 1996, ApJ, 459, 298

Cassisi, S., Castellani, M., \& Castellani, V. 1997, A\&A, 317, 108

Cordier, S., Pietrinferni, A., Cassisi, S., \& Salaris, M. 2007, ApJ, 133, 468 (Paper III)

Cox, J. P., \& Giuli, R. T. 1968, Principles of stellar structure (London: Gordon \& Breach)

Demarque, P., Woo, J.-H., Kim, Y.-C., \& Yi, S. K. 2004, ApJS, 155, 667

Dotter, A., Chaboyer, B., Ferguson, J. W., et al. 2007, ApJ, 666, 403

Ferguson, J. W., Alexander, D. R., Allard, F., et al. 2005, ApJ, 623, 585

Fulbright, J. P., Rich, R. M., \& Castro, S. 2004, ApJ, 612, 447

Gonzalez, O. A., Rejkuba, M., Zoccali, M., Valenti, E., \& Minniti, D. 2011, A\&A, 534, A3

Gratton, R., Sneden, C., \& Carretta, E. 2004, ARA\&A, 42, 385

Greggio, L., \& Renzini, A. 1990, ApJ, 364, 35

Grevesse, N., \& Noels, A. 1993, in Origin and Evolution of the Elements, eds. N. Prantzos, E. Vangioni-Flam, \& M. Cassé (Cambridge: Cambridge Univ. Press), 14

Grevesse, N., \& Sauval, A. J. 1998, Space Sci. Rev., 85, 161

Krishna-Swamy, K. S. 1966, ApJ, 145, 174

Kuehn, C., Kinemuchi, K., \& Ripepi, V., et al. 2008, ApJ, 674, L81

Kunz, R., Fey, M., Jaeger, M., et al. 2002, ApJ, 567, 643

Norris, J. E., Gilmore, G., Wyse, R. F. G., et al. 2008, ApJ, 689, L113

Norris, J. E., Yong, D., Gilmore, G., \& Wyse, R. F. G. 2010, ApJ, 711, 350

Norris, J. E., Yong, D., Bessell, M. S., et al. 2013, ApJ, 762, 28

Percival, S. M., Salaris, M., Cassisi, S., \& Pietrinferni, A. 2009, ApJ, 690, 427 (Paper IV)

Pietrinferni, A., Cassisi, S., Salaris, M., \& Castelli, F. 2004, ApJ, 612, 168 (Paper I)

Pietrinferni, A., Cassisi, S., Salaris, M., \& Castelli, F. 2006, ApJ, 642, 797 (Paper II)

Pietrinferni, A., Cassisi, S., Salaris, M., Percival, S., \& Ferguson, J. W. 2009, ApJ, 697, 275 (Paper V)

Raimondo, G., Brocato, E., \& Cassisi, S. 2001, Mem. Soc. Astr. It., 72, 747

Reimers, D. 1975, Mem. Soc. R. Sci. Liège, 8, 369

Salaris, M., Cassisi, S., Pietrinferni, A., Kowalski, P. M., \& Isern, J. 2010, ApJ, 716, 1241 (Paper VI)

Sweigart, A. V., Greggio, L., \& Renzini, A. 1989, ApJS, 69, 911

Sweigart, A. V., Greggio, L., \& Renzini, A. 1990, ApJ, 364, 527 